\title{
Synaptic interactions increase optic flow specificity
}

\author{
Wolfram Horstmann, Martin Egelhaaf and Anne-Kathrin Warzecha \\ Lehrstuhl für Neurobiologie, Fakultät für Biologie, Universität Bielefeld, Postfach 1001 31, D-33501 Bielefeld, Germany
}

Keywords: fly, motion vision, neural coding, reliability

\begin{abstract}
Representations of optic flow are encoded in fly tangential neurons by pooling the signals of many retinotopically organized local motion-sensitive inputs as well as of other tangential cells originating in the ipsi- and contralateral half of the brain. In the so called HSE cell, a neuron involved in optomotor course control, two contralateral input elements, the $\mathrm{H} 1$ and $\mathrm{H} 2$ cells, mediate distinct EPSPs. These EPSPs frequently elicit spike-like depolarizations in the HSE cell. The synaptic transmission between the H2 and the HSE cell is analysed in detail and shown to be very reliable with respect to the amplitude and time-course of the postsynaptic potential. As a consequence of its synaptic input, the HSE cell responds best to wide-field motion, such as that generated on the eyes when the animal turns about its vertical body axis. It is shown that the specificity of the HSE cell for this type of optic flow is much enhanced if rapid membrane depolarizations, such as large-amplitude EPSPs or spike-like depolarizations, are taken into account rather than the average membrane potential.
\end{abstract}

\section{Introduction}

Whenever an animal is moving around in its environment, the retinal images are displaced in a way characteristic of the direction and velocity of the animal's self-motion and of the three-dimensional layout of the surround. Consequently, this so-called optic flow provides information for visually guided orientation. Neurons sensitive to particular aspects of optic flow have been characterized in a variety of species, ranging from fast moving insects (e.g. Collett \& King, 1975; Hausen \& Egelhaaf, 1989; Kern, 1998; Egelhaaf \& Warzecha, 1999; Rind \& Simmons, 1999) to various vertebrates (e.g. Duffy, 1998; Sun \& Frost, 1998; Wylie et al., 1998). The motionsensitive tangential cells (TCs) of the blowfly are amenable to experimental analysis of the cellular computations underlying the specific sensitivities to optic flow (Hausen \& Egelhaaf, 1989; Egelhaaf \& Borst, 1993a; Hengstenberg etal., 1998; Egelhaaf \& Warzecha, 1999). The specificity of TCs to optic flow is attributed to two types of synaptic input. (i) They pool with their extended dendrites the outputs of large numbers of retinotopically organized local motion sensitive elements. (ii) The TCs receive synaptic input from other TCs (Hausen, 1981; Hausen \& Egelhaaf, 1989; Egelhaaf etal., 1993; Warzecha et al., 1993). Here we analyse for a particular TC, the so called HSE cell, how the latter type of synaptic input affects the intrinsic properties of the cell and its specificity for optic flow.

The HSE cell (Hausen, 1982a,b) has been shown to play a role in optomotor course control (Heisenberg et al., 1978; Geiger \& Nässel, 1982; Hausen \& Wehrhahn, 1983; Egelhaaf, 1985, 1987; Bausenwein etal., 1986). It responds to front-to-back motion in the ipsilateral visual field with graded depolarizations which may be superimposed by spike-like depolarizations ('spikelets'). During ipsilateral back-tofront motion the HSE cell is hyperpolarized (Hausen, 1982a). The graded de- and hyperpolarizations are assumed to be the pooled postsynaptic signals of the retinotopically organized inputs (Fig. 1B).

Correspondence: Dr Anne-Kathrin Warzecha, as above.

E-mail: ak.warzecha@biologie.uni-bielefeld.de

Received 16 November 1999, revised 2 March 2000, accepted 3 March 2000
Due to the large length constant of the relatively thick axon of the HSE cell (Borst \& Haag, 1996), these graded stimulus-induced membrane potential changes are conducted to the cell's output terminal, suggesting that they may play a role in controlling transmitter release. The HSE cell receives excitatory input from the contralateral half of the brain during back-to-front motion. It was proposed that this input was mediated by two spiking TCs, the $\mathrm{H} 1$ and the H2 cell (Hausen, 1976; 1982a). The cellular morpholgy of these neurons suggests that the $\mathrm{H} 1$ cell connects to the main dendritic arborization of the HSE cell, whilst the signals of the $\mathrm{H} 2$ cell impinge on the HSE cell close to its output region (Fig. 1B).

Owing to its input organization the HSE cell should respond best to rotations about the fly's vertical body axis. Nonetheless, its average response to contralateral motion was found to be almost negligible, and its response to rotational optic flow to be not much larger than the response to translational optic flow (Hausen, 1982b). To resolve this puzzle, we studied the contralateral synaptic input with respect to its dynamic properties and its reliability. We show (i) that, owing to its transient nature, the contralateral input often elicits spikelets in the HSE cell and (ii) that, as a consequence of the contralateral input, the specificity of the HSE cell to rotational optic flow is much higher if the response transients, rather than the average membrane potential, are taken into account.

An abstract covering parts of this paper has been published previously (Horstmann etal., 1999).

\section{Materials and methods}

\section{Dissection of the animals and electrophysiological recording}

All experiments were done on blowflies of the genus Calliphora which were bred in our laboratory stocks. Animals were dissected following the routine for intracellular recording as described previously (Warzecha etal., 1993). Animal care was in accordance with institutional guidelines. Experiments were carried out in accordance with the European Communities Council Directive (86/ 609/EEC). Intracellular recordings were made with electrodes which 
were pulled from borosilicate glass (GC100F-10, Clark Electromedical, now: Harvard Apparatus Ltd, Kent, England) with a Flaming-Brown Puller (P97, Sutter Instrument, Novato, CA, USA). When filled with $1 \mathrm{M} \mathrm{KCl}$, electrodes had resistances between 30 and $60 \mathrm{M} \Omega$. Extracellular recordings were also performed with glass electrodes (GC100TF-15, Clark Electromedical) which, however, had a much lower resistance $(<5 \mathrm{M} \Omega)$. Recordings were made with standard electrophysiological equipment. The intracellular and extracellular signals were sampled at a rate of $2 \mathrm{kHz}$ each. The data were recorded and evaluated by programs written in $\mathrm{C} / \mathrm{C}^{++}$(Borland, now: Inprise Corporation, Scotts Valley, CA, USA) or ASYST (Keithley Instruments, Taunton, MA, USA). The HSE cell in the right half of the brain was recorded intracellularly close to its output site. The $\mathrm{H} 1$ and $\mathrm{H} 2$ cells which have their input region in the contralateral half of the brain were recorded extracellularly close to their respective output sites in the right half of the brain (see Fig. 1B). Cells were identified on the basis of their receptive field properties, preferred direction of motion, response modes and the recording site. No element with similar structural and physiological properties to the $\mathrm{H} 1$ cell has ever been described. It is thus generally assumed that the $\mathrm{H} 1$ cell can be identified unambigously on the basis of its functional properties in extracellular recordings. In contrast, at least two neurons with simiar functional properties and a similarly located output region as the $\mathrm{H} 2$ cell have been described (Strausfeld et al., 1995; Douglass \& Strausfeld, 1996). Therefore the identification of the H2 cell has to be regarded as tentative. Note, however, that in all double recordings of a tentative $\mathrm{H} 2$ and an HSE cell, every large and distinct excitatory postsynaptic potential (EPSP) in the membrane potential of the HSE cell was associated with a spike in the extracellular record. Moreover, we never observed spikes in an extracellularly recorded neuron tentatively characterized as an $\mathrm{H} 2$ cell that were not associated with EPSPs or spikelets (see below) in the HSE cell. We therefore will term this neuron the $\mathrm{H} 2$ cell irrespective of its anatomical properties. Animals were adjusted within the stimulus arena according to the symmetry of their eyes. In all experiments except the ones shown in Fig. 1A and Fig. 4B the activity of the HSE cell was recorded simultaneously with the activity of either the $\mathrm{H} 1$ or the $\mathrm{H} 2$ cell.

\section{Visual stimulation}

Two types of stimulus equipment were used for generating visual stimuli. In the experiments shown in Figs 1C 2, 3 and 4A, visual stimuli were generated on four boards each covered with an array of 48 columns and 30 rows of green LEDs $\left(5 \times 2.5 \mathrm{~mm}^{2}\right)$. The vertical columns of the LED arrays could be switched on or off independently. The LED boards were built in our departmental electronic workshop. They were arranged on a circular line covering a section along the azimuth from $-80^{\circ}$ to $+80^{\circ}$ with respect to the midline of the fly. In order to be able to stimulate exclusively the ipsior the contralateral eye, motion stimuli were not presented in an area between the azimuthal angles of $-20^{\circ}$ and $+20^{\circ}$, which well comprises the area of binocular overlap. The vertical extent of the pattern amounted to $\approx \pm 30^{\circ}$, with $0^{\circ}$ denoting the horizontal plane of the fly. Vertical square-wave gratings with a spatial wavelength of $13.3^{\circ}$ were used as motion stimuli. The brightness of the stimulus amounted to $21 \mathrm{~cd} / \mathrm{m}^{2}$ when the LEDs were switched on and to $0.01 \mathrm{~cd} / \mathrm{m}^{2}$ when they were switched off. During the first second of a stimulus sweep (total duration $5 \mathrm{~s}$ ) the grating was stationary. During the following two seconds, instantaneous displacements of the grating by one LED column were generated every $500 \mathrm{~ms}$. During the last two seconds apparent motion stimuli were presented at a velocity of
$83 \%$ by displacing the grating by the width of one LED column every $10 \mathrm{~ms}$. The direction of motion was either from the front to the back or from the back to the front and could be selected independently for each eye. By appropriate combination of ipsilateral and contralateral motion, different types of optic flow, such as rotation about the vertical axis of the fly as well as translation, were simulated.

In the experiments shown in Figs $1 \mathrm{~A}$ and 4B, optic flow stimuli were generated on two CRT screens (Tektronix 608, Wilsonville, OR, USA). The stimulus patterns were generated at a frame rate of approximately $200 \mathrm{~Hz}$ by two image synthesizers (Picasso, Innisfree; Cambridge, MA, USA). The monitor screens were placed symmetrically in front of the fly subtending an angle of $90^{\circ}$ and perpendicular to the horizontal plane of the eye. The centres of the screens were placed at an azimuth of $\pm 50^{\circ}$ and at an elevation of $0^{\circ}$. The horizontal and vertical extent of each pattern amounted to $58.5^{\circ}$ and $45^{\circ}$, respectively. The stimulus pattern was a squarewave grating with an angular wavelength of $12.7^{\circ}$ in the middle of the screens, a mean luminance of $8.2 \mathrm{~cd} / \mathrm{m}^{2}$ and a contrast of 20 or $35 \%$. Five different motion stimuli were presented at a velocity of $25.4 \%$ in a pseudorandom order, so that each stimulus was presented once before the next repetition started: contralateral back-to-front, contralateral front-to-back, ipsilateral front-to-back, contra- and ipsilateral front-to-back (simulating translatory optic flow), and contralateral back-to-front together with ipsilateral front-to-back (mimicking rotatory optic flow). During the first second of each stimulus sweep (total duration $3.5 \mathrm{~s}$ ) the resting activity was recorded. Then one of the five motion stimuli was presented for $2.5 \mathrm{~s}$. Subsequently, after an interval of $6 \mathrm{~s}$ without pattern motion, the next stimulus sweep started.

\section{Data analysis}

In all experiments except the ones shown in Figs 1A and 4B one data set consisted of 10 sweeps; $4900 \mathrm{~ms}$ of the sweep starting $50 \mathrm{~ms}$ after its onset were evaluated. During contralateral motion EPSPs in the HSE cell which are induced by the $\mathrm{H} 2$ neuron can be clearly distinguished by their large size and characteristic time course (see below). Therefore, it was not necessary to simultaneously record the activity of the $\mathrm{H} 2$ and the HSE cell to identify the H2-induced EPSPs, as long as the EPSPs did not have a spikelet superimposed. If not stated otherwise double recordings between H1 and HSE cells during stimulation with back-to-front motion on the contralateral side were taken for analysis. In these data sets H1-induced EPSPs were identified by their fixed temporal relationship to the H1 spikes. Spikelets and H2 EPSPs in the HSE signal were detected by searching for transient membrane potential changes of $>+3 \mathrm{mV} / \mathrm{ms}$. Spikelets and H2 EPSPs were distinguished by their peak amplitude $(>20 \mathrm{mV}$ for spikelets). A spike in the $\mathrm{H} 2$ cell was found to precede by $0.5 \mathrm{~ms}$, on average, the beginning of the EPSP, i.e. the time bin after which the membrane potential increased by $>3 \mathrm{mV} / \mathrm{ms}$. With this procedure it was possible to identify both $\mathrm{H} 1$ - and $\mathrm{H} 2$-induced EPSPs in double-recordings of the H1 and the HSE cell and, hence, to avoid triple recordings. The procedure of detecting H2-induced EPSPs was tested by cross-correlating measured $\mathrm{H} 2$ spikes obtained from double recordings of the $\mathrm{H} 2$ and HSE cells with spikes which were reconstructed from the occurrence of H2-induced EPSPs determined as described above. Ninety-five percent of the reconstructed $\mathrm{H} 2$ spikes were in the same time-bin as the measured $\mathrm{H} 2$ spikes and $5 \%$ showed a jitter of one time bin (i.e. $\pm 0.5 \mathrm{~ms}$ ). The reconstruction procedure was only feasible if the H2 EPSPs did not elicit spikelets. Therefore, spikelets were excluded from parts of the analysis. 


\section{Results}

The HSE cell responded to optic flow with a different signal form depending on the stimulated eye and the direction of motion (Fig. 1A; Hausen, 1982a). Ipsilateral front-to-back motion led to a graded depolarizing shift of the membrane potential on which were superimposed relatively slow membrane potential fluctuations as well as fast, spike-like depolarizations ('spikelets'). The responses looked quite similar during bilateral front-to-back motion simulating translatory optic flow. In contrast, during contralateral back-to-front motion the cell was not much depolarized. Instead, short and rapid depolarizations of different amplitudes could be observed. During clockwise rotatory motion simulating counterclockwise rotation of the animal about its vertical body axis, these rapid depolarizations superimpose graded depolarizations. The rapid depolarizations are either EPSPs elicited by the $\mathrm{H} 2$ or $\mathrm{H} 1$ cell, respectively, or spikelets. The origin of the EPSPs could be identified by double recordings of either the $\mathrm{H} 2$ or $\mathrm{H} 1$ cell and the HSE cell and by using relatively weak motion stimuli, i.e. stimuli that did not lead to responses where the various signals superimpose too much (Fig. 1C). Whenever the $\mathrm{H} 2$ or $\mathrm{H} 1$ cell generated an action potential, a distinct EPSP was induced in the HSE cell. The EPSPs induced by the $\mathrm{H} 2$ cell were generally larger than those induced by the $\mathrm{H} 1$ cell. Depending on the strength of motion stimulation, the EPSPs appeared to elicit occasionally a spikelet (see arrows in Fig. 1C). During contralateral back-to-front motion when there were no membrane potential fluctuations due to ipsilateral synaptic input, the H2-induced EPSPs were quite large and could be identified unambiguously. A similar connection has been established on the basis of double recordings between the H2 and the HSN cell (Haag, 1994; Haag et al., 1999). In the latter contribution (Haag etal., 1999) the HSN cell has been labelled HSE cell by mistake (Haag, personal communication). In the following, three aspects will be addressed. (i) The H1- and H2induced EPSPs and their potential interactions are characterized. (ii) The reliability of synaptic transmission is analysed for the connection between the H2 and the HSE cell. (iii) The consequences of the

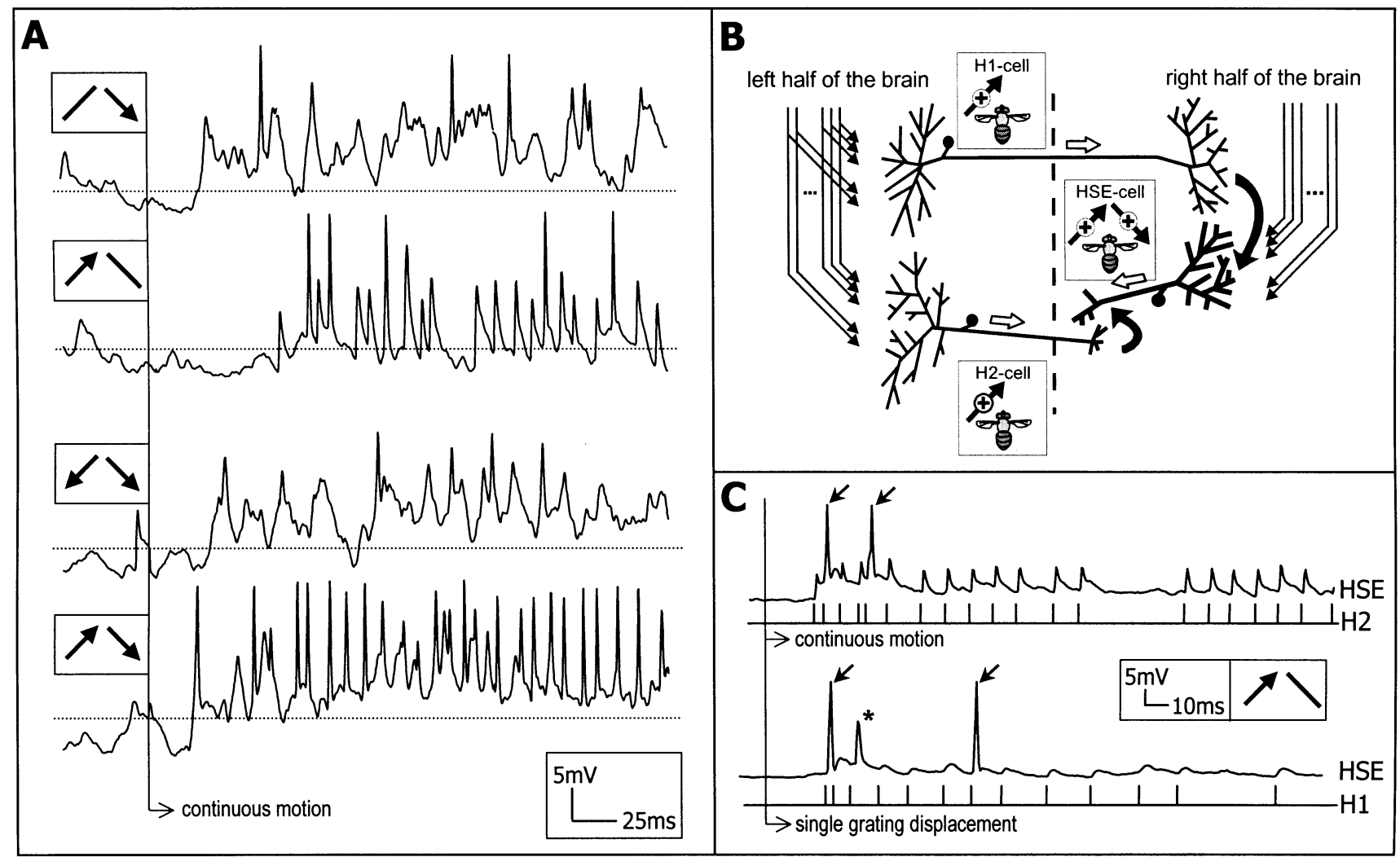

FIG. 1. Response characteristics and input organization of HSE cell. (A) Timecourses of responses of an HSE cell in the right half of the brain to various types of optic flow, i.e. translational and rotational optic flow, as well as their corresponding monocular components. The arrows indicate front-to-back and back-to-front motion in the left and right visual field, respectively. Whilst the cell mainly shows graded depolarizations during ipsilateral front-to-back motion (first trace) and translational optic flow (third trace), there are many response transients during contralateral back-to-front motion (second trace) and clockwise rotational optic flow (fourth trace). The resting potential is indicated by horizontal dotted lines, the motion onset by the thin vertical line. Pattern contrast, 35\%. (B) Schematic of synaptic input organization of the right HSE cell. The HSE cell receives input from many retinotopically organized local motion-sensitive input elements (indicated by thin lines) in the ipsilateral visual field. Information about back-to-front motion in the contralateral visual field is mediated by the $\mathrm{H} 1$ and the $\mathrm{H} 2 \mathrm{cell}$ (thick black arrows). The $\mathrm{H} 2$ cell contacts the HSE cell close to its output terminal and the $\mathrm{H} 1$ cell probably makes a multitude of synaptic connections with its extended terminal region on the dendritic tree of the HSE cell. Open arrows indicate the direction of signal flow. Insets illustrate, seen from above, the fly looking at various motion stimuli and indicate the preferred directions of motion of the different cells. (C) Upper diagram, double recording of an HSE and an $\mathrm{H} 2$ cell. The time-dependent membrane potential of the HSE cell and the time of occurrence of extracellularly recorded spikes of the H2 cell are plotted. The cells were stimulated by motion of the contralateral stimulus pattern from the back to the front. The onset of stimulation is indicated by the long vertical line. Lower diagram, double recording of an HSE and an H1 cell. Otherwise, as in the recording shown in the upper diagram. Responses were elicited by a single displacement of the contralateral stimulus pattern from the back to the front. This stimulus condition was used because most of the H1-induced EPSPs have large depolaristions superimposed under the other stimulus conditions and are hence not easily visible (see upper diagram). The large EPSP (asterisk) is most probably elicited by a spike in the $\mathrm{H} 2$ cell (see Materials and Methods). The different sizes of the different types of EPSPs in the upper and lower HSE response reflect the variability found between different cells (see text). Spikelets are marked by arrows. 
contralateral synaptic input of the HSE cell is investigated with respect to the specificity of the HSE cell to rotational optic flow as it is experienced by an animal turning around its vertical body axis.

\section{Characterization of EPSPs elicited by contralateral motion}

The H1- and H2-induced EPSPs differed in both their amplitude and their time course. The peak amplitude of the mean H1-induced EPSP
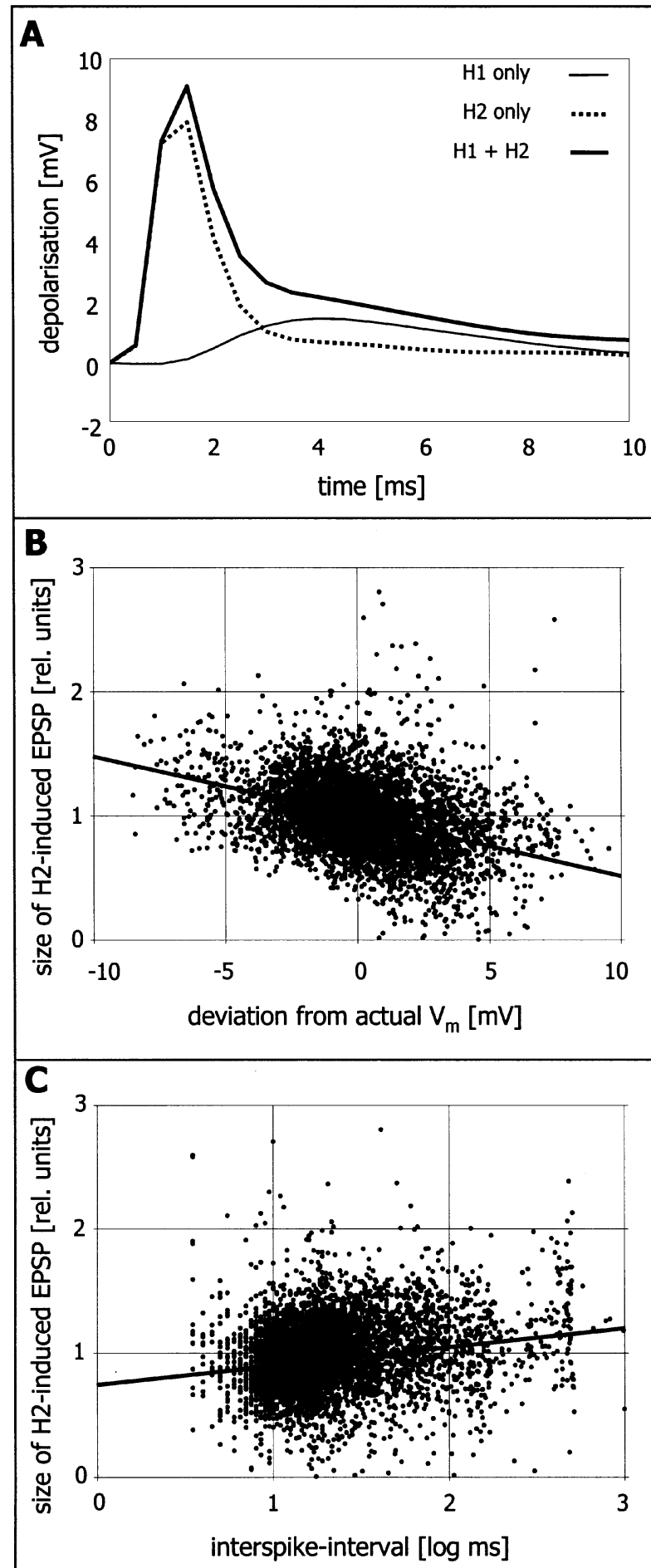

varied for different cells between 1.5 and $2.0 \mathrm{mV}$ (mean $1.8 \mathrm{mV}$; $N=4$ cells). The amplitude of the mean $\mathrm{H} 2$-induced EPSP was found to be much larger and varied for different cells between 5.3 and $11.9 \mathrm{mV}$ (mean $8.7 \mathrm{mV}, N=5$ cells). The H2-induced EPSPs were much faster than the H1-induced EPSPs (Fig. 2A). The different dynamic properties of both EPSP types was reflected in different risetimes and time-constants of decay. Whilst the rise-time, determined as the time it takes the EPSP to depolarize from 10 to $90 \%$ of its maximum, lasted between 1.8 and $2.1 \mathrm{~ms}$ for the mean $\mathrm{H} 1$-induced EPSP ( $N=4$ cells), it ranged for the mean H2-induced EPSP only between 0.7 and $0.8 \mathrm{~ms}(N=5$ cells $)$. The decay time from the peak response to $37 \%$ of this value varied for the mean H1-induced EPSP between 4.6 and $6.1 \mathrm{~ms}(N=4$ cells), whereas it ranged between 0.9 and $2.0 \mathrm{~ms}$ for the mean H2-induced EPSP ( $N=5$ cells). Parameters of the EPSPs' time course were approximated for each cell separately from a linear interpolation between the data points of the mean EPSPs.

The $\mathrm{H} 1$ and $\mathrm{H} 2$ cells tend to fire in synchrony with a timeshift between spikes in the order of only 1-2 ms, although at different frequencies (Warzecha et al., 1998). Therefore, it was interesting to find out whether the two types of EPSPs superimpose linearly or interact in a nonlinear way. This question was approached by double recordings between the $\mathrm{H} 1$ and the $\mathrm{HSE}$ cell, when the $\mathrm{H} 2$-induced EPSPs were identified on the basis of their amplitude and timecourse in the membrane potential of the HSE cell (see Materials and Methods). The amplitude and timecourse of H2-induced EPSPs when they coincided with a spike in the $\mathrm{H} 1$ cell (i.e. $\mathrm{H} 2$ and $\mathrm{H} 1$ cell fired synchronously) were compared with H2-induced EPSPs when there was no synchronous $\mathrm{H} 1$ spike. The joint $\mathrm{H} 1 / \mathrm{H} 2$-induced EPSP was found to be only slightly larger than the H2-induced EPSP, as would be expected if the two types of EPSP superimpose about linearly (Fig. 2A). Although this linearity is difficult to infer from the timecourse of the EPSPs, the decay of the joint $\mathrm{H} 1 / \mathrm{H} 2$-induced EPSP appears to be somewhat slower than the decay of the H2-induced EPSPs alone. A detailed quantitative comparsion of the interactions

FIG. 2. Characteristics of H1- and H2-induced EPSPs. The data used for this analysis were obtained during contralateral stimulation with single displacements of the stimulus pattern and motion stimuli from back-to-front (see Materials and Methods). (A) Mean EPSPs elicited in the HSE cell by spikes in the $\mathrm{H} 1$ and the $\mathrm{H} 2$ cell. Thin line, only $\mathrm{H} 1$ cell active; dotted line, only $\mathrm{H} 2$ cell active; thick line, $\mathrm{H} 1$ and $\mathrm{H} 2$ cell simultaneously (within $\pm 1 \mathrm{~ms}$ ) active. The amplitude of the joint H1/H2 EPSP is slightly larger than that of the EPSP elicited by the H2 cell alone. Measured values are connected by straight lines. Only those EPSPs which met the following condition were included in the analysis. Neither a spikelet nor another EPSP than the one under investigation was allowed to occur in the HSE cell response within a time interval of $20 \mathrm{~ms}$ centred around the onset of the analysed EPSP. The H2 signal was inferred from the postsynaptic signal (see Materials and Methods). Time ' 0 ' indicates the occurrence of a spike in the H1 cell (thin line) or a reconstructed spike in the $\mathrm{H} 2$ cell (thick and dotted lines). Mean over 247 events (thin line), 44 events (dotted line) and 400 events (thick line) obtained in a single recording of the H1 and the HSE cell. Similar results were obtained in three other recordings. (B) EPSP size as a function of membrane potential. The membrane potential is given as the deviation from its average resting level. The size of the EPSP induced by the $\mathrm{H} 2$ cell was determined within a time interval of $5 \mathrm{~ms}$ relative to the membrane potential at the time of onset of the EPSP. (C) EPSP size as a function of the interspike interval between consecutive $\mathrm{H} 2$ spikes. Note that owing to the discrete sampling times interspike intervals could only assume integral multiples of $0.5 \mathrm{~ms}$. (B and C) The size of EPSPs induced by the $\mathrm{H} 2$ cell was determined irrespective of whether a H1spike occurred. Data were obtained from five preparations. The mean EPSP size of each preparation was normalized to 1 . The tilted thick lines are the average regression lines of five cells. 
between H1- and H2-induced EPSPs was not possible, because of their very unequal size. In any case, there are no conspicuous nonlinearities when both types of EPSPs are elicited simultaneously.

The amplitude of postsynaptic potentials of many synaptic connections depends systematically on parameters such as the membrane potential or the interval between the presynaptic spikes. Moreover, the synaptic gain is often quite plastic (see, e.g., Murthy, 1999). Therefore, it was investigated whether or not such factors affect the amplitude of the H2-induced EPSPs. This analysis was possible without manipulating the membrane potential of the HSE cell by current injection via the electrode, because the membrane potential was not constant, but modulated during stimulation. EPSPs of the $\mathrm{H} 1$ and $\mathrm{H} 2$ cell are superimposed on these membrane potential fluctuations. Since the determination of the exact EPSP amplitude is prone to inaccuracies caused by the discrete sampling of the membrane potential, the mean amplitude was determined over a 5ms interval instead of the peak amplitude. The $5 \mathrm{~ms}$ interval started with the transient depolarization of the membrane potential as specified in Materials and Methods. This time-averaged amplitude is termed, for convenience, EPSP size. A regression analysis of the data suggested that the EPSP size decreased slightly with increasing depolarization of the HSE cell (on average, by $0.05 \mathrm{mV}$ per $\mathrm{mV}$ of membrane potential change; linear regression: $n=4648, N=5$ cells, $R^{2}=0.115, P<0.001$, Fig. 2 B). Such a dependence is, at least qualitatively, expected when the EPSP size is mainly determined by the driving forces of the underlying currents. The same conclusion has been reached by Haag etal. (1999) for HS cells that were hyperpolarized by current injection during contralateral motion stimulation. In addition to the dependence on membrane potential, the size of H2-induced EPSPs slightly increased with increasing interspike interval (factor of 0.14 per log ms of the interspike interval; linear regression: $n=4648 ; \quad N=5$ cells, $R^{2}=0.035, \quad P<0.001$,

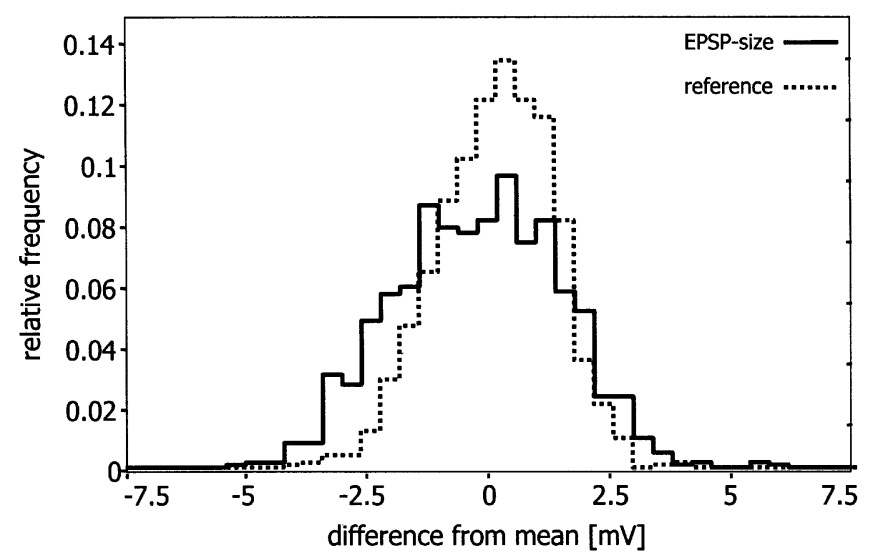

FIG. 3. Distribution of the size of the EPSPs induced by the $\mathrm{H} 2$ cell. The distribution indicates the probability with which a given EPSP size deviates from the mean EPSP size. As a reference, the membrane potential noise was determined by subtracting the membrane potential at the onset of each $\mathrm{H} 2$ spike from the corresponding averaged membrane potential within $5 \mathrm{~ms}$ prior to the spike. Data derive from a double recording of the HSE and the $\mathrm{H} 2$ cell. Similar results were obtained from four other recordings of an HSE cell. If, $10 \mathrm{~ms}$ before or after an $\mathrm{H} 2$-induced EPSP, spikelets or further $\mathrm{H} 2$-induced EPSPs were observed in the response of the HSE cell, the corresponding values of the EPSP size and the reference were excluded from analysis. Since the $\mathrm{H} 1$ and the $\mathrm{H} 2$ cell often generate spikes synchronously, applying this same procedure to H1-induced EPSPs made it not feasible to obtain enough data for the probability distributions. Therefore, the variability of the EPSPs as well as that of the reference might have been slightly overestimated. Data were recorded during stimulation with contralateral back-to-front motion.
Fig. 2C). The actual membrane potential of the HSE cell at the onset of the EPSP slightly depolarized when the $\mathrm{H} 2$ spikes closely followed each other (factor of 1.18 per log ms of the interspike interval; linear regression: $n=4648, N=5$ cells, $R^{2}=0.031, P<0.001$, not shown). This depolarization has its likely origin in a superposition of subsequent EPSPs when they are elicited in close succession. Hence, the dependence of the EPSP size on the interspike interval of the $\mathrm{H} 2$ cell probably reflects the membrane potential dependence of the EPSP amplitude rather than synaptic plasticity.

In conclusion, the EPSPs elicited by the $\mathrm{H} 2$ cell showed neither enhancement nor depression in dependence of their frequency of occurrence; rather the EPSP size appeared to be essentially determined by the driving forces of the underlying postsynaptic currents. A similar analysis was not possible for the H1-induced EPSPs because of their small size and because they were superimposed frequently by the much larger H2-induced EPSPs.

\section{Reliability of synaptic transmission}

As is known for many types of synaptic connections, synapses can be very unreliable and thus may constrain to a large extent the reliability with which information is transmitted by the nervous system (see, e.g. Calvin \& Stevens, 1968; Murthy, 1999). Therefore, we analysed the reliability of the H2-HSE synapse. Because of the small size of the H1-induced EPSP a similar analysis was not performed for this synaptic connection. Whenever a spike of the $\mathrm{H} 2$ cell was fired, a large EPSP or a spikelet, which most probably was elicited by the EPSP (see below), could be recorded in the HSE cell. Hence there was no indication of synaptic failure. Nonetheless the EPSP size was found to vary to some extent. The distribution of EPSP size is approximately Gaussian (see solid line in Fig. 3 for an example; standard deviation of EPSP size $1.97 \mathrm{mV}, N=5$ cells). This variability cannot be interpreted as variability of synaptic transmission alone, because the EPSP size is affected by two other factors. (i) The membrane potential of the HSE cell on which H2 EPSPs superimpose was found to systematically influence EPSP size (Fig. 2B). Therefore, the variability of EPSP size was also determined after correcting for the membrane potential dependence of the EPSP size. When the EPSP size was scaled according to the slope of the regression line characterizing the relationship between EPSP size and membrane potential, the distribution of EPSP size (standard deviation of corrected EPSP size $1.87 \mathrm{mV}, N=5$ cells) was somewhat narrower than without this correction. Hence, a small part of the total observed EPSP size variability can be attributed to a systematic dependence of the EPSP size on the membrane potential. (ii) Even without H2induced EPSPs the membrane potential of the HSE cell does not stay constant within the 5-ms time interval used to determine EPSP size. Therefore the distribution of the background noise was also determined. This was done in the following way. The membrane potential at the onset of the $\mathrm{H} 2$ spike was subtracted from the average membrane potential within a 5 -ms time interval prior to the onset of the $\mathrm{H} 2$ spike. This was done for each $\mathrm{H} 2$-induced EPSP. The resulting distribution was used as a reference distribution (dotted line in Fig. 3, standard deviation of background noise $1.31 \mathrm{mV}, N=5$ cells). A correction of background noise for membrane potential dependence as has been done above for EPSP size is not appropriate, because background noise cannot be expected to show a similar dependence on membrane potential as this noise arises from depolarizing as well as hyperpolarizing events. The finding that the distribution of the corrected EPSP sizes is broader than that of the background noise indicates that synaptic transmission contributes some variability to EPSP size. 


\section{W. Horstmann et al.}

In order to assess the contributions of the background noise and the synaptic noise to the EPSP size variability, the following approximations were made. Assuming that the EPSPs add linearly to the actual membrane potential and that, consequently, both noise components do so as well, the standard deviation of the synaptic noise can be calculated. It is given by the square root of the difference of the variances of the EPSP size and of the background noise. If approximated in this way, we obtain for the synaptic transmission variability a standard deviation of $1.33 \mathrm{mV}$ (after correction for membrane potential dependence of EPSP size, $N=5$ cells). Hence, on average, both synaptic noise and background noise contribute about equally to the variability of the H2-induced EPSPs. A relative contribution in a similar range was recently found for another synapse in the insect visual pathway (Simmons, 1999). On their own, these measures do not tell much about the reliability of synaptic transmission. Rather, they have to be related to the size of the EPSPs. Therefore, the signal-to-noise ratio, i.e. the ratio between the standard deviation and the EPSP size, was calculated. For the overall EPSP size including the background noise, the signal-to-noise ratio amounted to 3.89 ( $N=5$ cells). The synaptic transmission between the $\mathrm{H} 2$ neuron and the HSE cell operates with a signal-to-noise ratio of 5.24 ( $N=5$ cells) when the synaptic noise is estimated as explained above. These findings thus indicate that spikes in the $\mathrm{H} 2$ cell and thus information about motion in the contralateral visual field are transmitted reliably to the postsynaptic neuron.

\section{Specificity for rotational optic flow}

What is the significance of the properties of the contralateral input of the HSE cell for tuning this cell to optic flow? We attempt to answer this question in two ways, (i) by analysing the relationship between the EPSPs elicited during contralateral motion and the generation of spikelets by the HSE cell, and (ii) by scrutinizing the timecourse of the HSE cell responses induced by different types of optic flow.

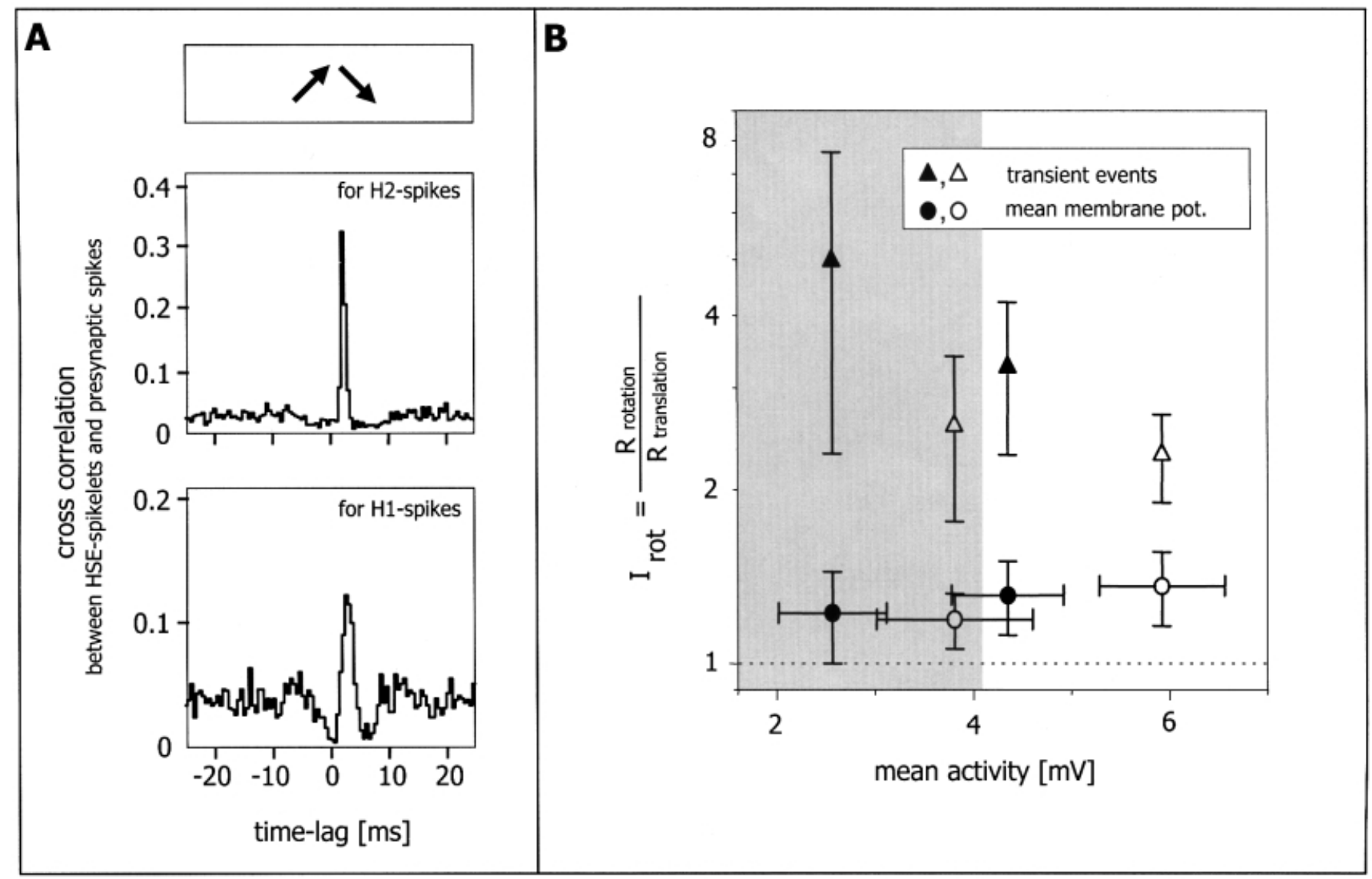

FIG. 4. Transient and graded responses elicited by optic flow. (A) Crosscorrelograms of spikes in the H2 cell (upper trace) or H1 cell (lower trace) and spikelets obtained in a single HSE cell during the stimuli mimicking rotational optic flow (see Material and Methods). Data are normalized to the total number of EPSPs of either the $\mathrm{H} 2$ cell or the $\mathrm{H} 1$ cell. Spikes in the H2 cell were recorded simultaneously with the activity of the HSE cell and were not reconstructed from EPSPs. Note the different ordinate scales. (B) Specificity of the HSE cell for rotational optic flow for a range of stimulus conditions plotted as a function of the mean membrane potential elicited by the respective stimulus condition. The stimulus pattern had either a contrast of $35 \%$ (white background) or 20\% (shaded background). Both the responses during the early response phase (averaged over the first $450 \mathrm{~ms}$ starting $50 \mathrm{~ms}$ after the onset of motion, open symbols) and the late response phase (averaged over $2000 \mathrm{~ms}$ starting $500 \mathrm{~ms}$ after the onset of motion, filled symbols) were analysed. The mean membrane potential and the number of transient events were determined in these time intervals. The mean membrane potential was calculated as the difference between the membrane potential and the resting potential. The resting potential was determined during $1 \mathrm{~s}$ prior to stimulus presentation. Membrane potential changes of at least $4 \mathrm{mV} / \mathrm{ms}$ were counted as transient events. The minimum interval between successive transient events was assumed to be $3 \mathrm{~ms}$. For each condition and cell a specificity index ( $\mathrm{I}_{\text {rot }}$ ), i.e. the ratio between the responses $(\mathrm{R})$ to rotational and translational optic flow were determined for both response modes. Values of $\mathrm{I}_{\text {rot }}>1$ indicate a greater specificity for rotational optic flow than for translational flow, values $<1$ a smaller specificity. Triangles indicate the specificity index values obtained for transient events; dots denote rotational specificity obtained for the mean membrane potential. Data are averages from eight cells (35\% contrast) and from six cells (20\% contrast). Five of these cells were stimulated with the high and with the low contrast. Error bars denote SEMs. Between 5 and 17 responses to identical motion stimulation were obtained from each cell. 
When cross-correlating the occurrence of $\mathrm{H} 2$ spikes and HSE spikelets it is obvious that the HSE spikelets are elicited to a large degree slightly delayed from $\mathrm{H} 2$ spikes and thus are most probably elicited by the resulting EPSPs. This is true whenever the $\mathrm{H} 2$ neuron fires, as is the case during contralateral back-to-front motion. During rotational optic flow about $60 \%$ of all HSE spikelets are generated within $2 \mathrm{~ms}$ after the onset of an $\mathrm{H} 2$ spike (Fig. 4A). Similar results are obtained when the occurrence of HSE spikelets are crosscorrelated with the occurrence of $\mathrm{H} 1$ spikes, although the peak in the cross-correlogram is smaller and slightly broader (Fig. 4A). Since the $\mathrm{H} 1$ and $\mathrm{H} 2$ cells do not fire independently, but tend to synchronize (Warzecha et al., 1998), it is not possible to assess their contribution to eliciting an HSE spikelet independently. In any case, HSE spikelets can be shown to be elicited during contralateral motion mainly as a consequence of the activity of the $\mathrm{H} 2$ and/or the $\mathrm{H} 1$ cell (see also Hausen, 1982a).

These results indicate that whenever there is back-to-front motion in the contralateral visual field, the responses of the HSE cell contain many transient membrane potential changes, representing either the relatively large H2-induced EPSPs or the spikelets. In contrast, when there is ipsilateral front-to-back motion there is a pronounced graded depolarization of the cell due to the many superimposed smallamplitude EPSPs elicited by the large number of retinotopic inputs (see Fig. 1A). For this reason, both the mean response amplitude and the number of response transients were evaluated separately and inspected with respect to their specificity for rotational optic flow. As response transients all membrane potential changes were counted during the period of motion stimulation which had a positive slope of at least $4 \mathrm{mV} / \mathrm{ms}$. This criterion slope is not critical, because similar results were obtained for a range of criteria for detecting response transients (e.g. $5 \mathrm{mV} / \mathrm{ms}$ for at least $0.5 \mathrm{~ms} ; 3 \mathrm{mV} / \mathrm{ms}$ for at least $1 \mathrm{~ms}$ ). The mean responses were determined similarly as in previous studies on the HSE cell (Hausen, 1982b) by averaging the response amplitude during an extended period of motion stimulation. To quantify the specificity of either response mode for rotational optic flow, a specificity index was determined as the ratio of the responses to rotational and translational optic flow for the mean and the transient responses separately. This was done for the responses obtained with two different pattern contrasts as well as for the early and the late phase of the responses (for definition of early and late response phase see legend of Fig. 4). These two response phases were evaluated separately, because motion-induced responses tend to decrease towards a final steady-state level after an initial response peak elicited directly after the onset of motion (Maddess \& Laughlin, 1985; Egelhaaf \& Borst, 1989). The specificity index was only slightly $>1$ when the mean membrane potential was evaluated. However, when the response transients were analysed, the specificity index increased significantly for all conditions (Fig. 4B, $P<0.025$, Wilcoxon's signed rank test for paired observations). Hence it can be concluded that, independent of the exact stimulus conditions, the specificity for rotational optic flow compared with translational optic flow is considerably higher when the response transients rather than the mean responses are taken into account.

\section{Discussion}

The sensitivity of the TCs of the fly to optic flow patterns elicited by self motion is obtained in two ways. On the one hand, TCs pool with their extended dendrites large numbers of retinotopically organized local motion-sensitive inputs. On the other hand, the specificity of the TCs to a particular type of optic flow may be further enhanced by input from the contralateral eye. Here we have provided evidence for the earlier suggestion (Hausen, 1976; 1982a) that one of the TCs, the HSE cell, receives its contralateral input from two TCs, the H1 and the $\mathrm{H} 2$ cell (Fig. 1C). Spikes in the $\mathrm{H} 2$ cell reliably elicit large postsynaptic potentials, with amplitudes ranging between $5 \mathrm{mV}$ and almost $12 \mathrm{mV}$ close to the output terminal of the HSE cell (Fig. 2A). The postsynaptic potentials induced by the $\mathrm{H} 1$ cell are much smaller (between 1.5 and $2 \mathrm{mV}$ ) than those elicited by the $\mathrm{H} 2$ cell, at least when recorded close to the output terminal of the HSE cell. The H1induced EPSPs may be much larger in the dendrites of the HSE cell where, for anatomical reasons, the synaptic contacts are to be expected. Since the EPSPs elicited by both contralateral input elements of the HSE cell are distinct and relatively brief they contribute only little to the average membrane potential (Hausen, 1982b; Fig. 4B). The specificity for rotational optic flow is much enhanced when the neural responses are not decoded on the basis of their average amplitude but rather when the frequency of rapid depolarizations, such as large EPSPs or spikelets, are taken into account (Fig. 4B).

In the following three questions will be adressed. (i) How are the postsynaptic signals integrated and how reliably is this done? (ii) How could the response transients be decoded from the overall neural responses and computationally separated from the much slower graded potential changes. (iii) What is the significance of binocular interactions for the specificity of visual interneurons to optic flow?

\section{Reliability of synaptic transmission and integration of synaptic input}

The synapse between the $\mathrm{H} 2$ cell and the HSE cell was found to be very reliable, at least when compared with many cortical synapses (see, e.g. Murthy, 1999). No synaptic failure was ever observed at the H2-HSE synapse, and the synapse was found to transmit presynaptic information with a large amplitude and a high signal-to-noise ratio of about 5 . The high reliability of the synaptic connection is probably due to its specific structural design. Although there is no ultrastructural study on this connection so far, such a specific design is not unlikely because it has been found for another TC, the VCH cell. This cell reveals a multitude of postsynaptic specializations in the region where it receives input from the $\mathrm{H} 2$ neuron (Gauck et al., 1997). It has not been possible to analyse the reliability of the H1HSE connection because of the relatively small size of the H1induced EPSPs. Likewise, for the retinotopic inputs of the HSE cell, it was not possible so far to single out an individual synaptic connection and to analyse its transmission properties.

The EPSP size only slightly decreased when the cell was depolarized due to other input or previous EPSPs. This slight decrease is most probably the consequence of a decrease in the driving forces of the postsynaptic currents. Accordingly, the superposition of $\mathrm{H} 1$ and $\mathrm{H} 2$ EPSPs which frequently occurs owing to the pronounced synchronicity of $\mathrm{H} 1$ and $\mathrm{H} 2$ cell firing (Warzecha et al., 1998), did not lead to an overproportional enhancement or depression of the joint EPSP. In contrast to synapses in many other systems (see, e.g., Koch, 1999; Murthy, 1999), the H2-HSE connection did not reveal any form of plasticity (Fig. 2). However, the activity of the H1 and/or H2 cells frequently elicits spikelets in the HSE cell (Fig. 4A; Hausen, 1982a). This finding is not surprising, because spikelets in the HSE cell are preferentially elicited by rapid membrane depolarisatons (Haag \& Borst, 1996).

\section{Possibilities for separating response transients and slow graded potential changes}

Cells mediating information by graded potential changes rather than by spike trains occur in particular in the peripheral sensory system. 
There are in addition cells where spike-like depolarizations are superimposed on the graded membrane potential changes (Roberts \& Bush, 1981). The significance of graded membrane potential changes vs. spikelets in fly visual interneurons is still a matter of debate (for review see Egelhaaf \& Warzecha, 1999). The present results suggest that more specific information about rotational optic flow can be decoded from the HSE response, if the rapid depolarizations rather than the graded membrane potential changes are taken into account. The latter, in contrast, basically represent front-to-back motion in the ipsilateral visual field largely independent of what is going on in the contralateral visual field. This finding indicates that different aspects of optic flow are encoded by graded membrane potential changes and by the rapid response transients.

Is it possible that postsynaptic neurons decode response transients separately from graded membrane potential changes? Although we do not know yet the functional properties of the neurons postsynaptic to the HSE cell, this may well be possible, depending on the dynamic properties of both the pre- and postsynaptic specializations. On the one hand, synapses have been characterized which transmit graded membrane potential changes by tonically releasing transmitter which can be up- and down-regulated depending on the presynaptic membrane potential (e.g. Roberts \& Bush, 1981; Juusola et al., 1995; Uusitalo et al., 1995; Manor et al., 1997). On the other hand, at many synapses rapid depolarizations, such as spikes, lead to transient calcium influx and transient transmitter release, thus boosting the transients rather than the graded potential changes (Bajjalieh, 1999; Koch, 1999; Murthy, 1999).

Although there is a debate about the functional significance of graded signalling vs. signalling by action potentials, both signal forms have been found to perform similarly in the visual system of the fly with respect to their capacity to represent motion information (for review see Egelhaaf \& Warzecha, 1999) as well as the energy which is consumed by the cell during information transfer (Laughlin et al., 1998). Now for the first time it has been shown that at least the HSE cell performs better in representing rotational optic flow when its transient response components rather than its graded ones are decoded by postsynaptic neurons.

\section{Significance of binocular interactions for the specificity of visual interneurons to optic flow}

The visual system of many moving animals exploits the information contained in the optic flow patterns generated on the retina during self-motion to control orientation behaviour. Biological mechanisms extracting information about retinal velocity are prone to imprecisions and noise (for review see, e.g., Egelhaaf \& Borst, 1993b) and therefore require the pooling of motion information from large parts of the visual field. It has been demonstrated that motion information from widely separated parts of the visual field of the two eyes interact to enlarge the specificity of rotational vs. translational optic flow (e.g. Insecta: Gaffron, 1934; Hertz, 1934; Hausen \& Egelhaaf, 1989; Ibbotson \& Goodman, 1990; Ibbotson, 1991; Kern \& Varjú, 1998; Kern, 1998; Decapoda: Kern etal., 1993; Columbiformes: Nalbach, 1992; Wylie \& Frost, 1999; Lagomorpha: Simpson et al., 1988). It has also been shown on theoretical grounds that motion information from opposite poles of the visual field are especially suitable for extraction of self-motion parameters from optic flow (Dahmen et al., 1997). Also, in flies, binocular interactions are known that increase the specificity of cells to rotational compared with translational optic flow (see Fig. 4B and for review: Hausen \& Egelhaaf, 1989; Egelhaaf \& Warzecha, 1999). The HSE cell that has been analysed in the present study is one of the output elements of the motion detection pathway of the fly. It connects via descending neurons to the flight motor centres in the thoracic ganglion (Strausfeld, 1976, 1989) and has been found to be involved in optomotor course control (see Introduction). Within the neuronal network involved in optomotor course control, the specificity to rotational optic flow is increased in a sequence of processing steps. (i) The distribution of the preferred directions of the local motion-sensitive elements, the signals of which are pooled by the TCs, are matched to flow fields as experienced by the animal during particular self-motion (Krapp \& Hengstenberg, 1996; Krapp etal., 1998). (ii) Information from the contralateral eye is added by synaptic connections from other TCs (Figs 1 and 2). Here the dynamic properties of the response play an important role for the specificity to rotation (Fig. 4). (iii) Finally, due to the bilaterally symmetrical body plan of the fly, the responses of the HSE cells in the right and the left half of the brain are compared at a later processing stage. Even if there should be no further interactions in the nervous system, the optic flow signals originating from both halves of the brain eventually interact at the behavioural level as a consequence of the mirror symmetrical input organization of the flight steering muscles (Heide, 1983; Egelhaaf, 1989). Hence, neuronal signals from the two visual hemifields converge twice in this sequence (steps ii and iii), thereby increasing the specificity of the pathway for rotational optic flow.

It is quite possible that the output signals of the HSE cell might be used twofold, although at present there is no evidence in this regard. If, in addition to the response transients, the graded membrane potential changes are decoded, aspects of optic flow others than the rotational component could be computed on the basis of the HSE cell output. As a consequence of the divergent stimulus-dependence of the graded and transient signal components, the HSE cell would then act as a kind of dual-information channel.

\section{Acknowledgements}

We thank Roland Kern, Holger Krapp and Rafael Kurtz for reading and critically discussing a previous version of this paper. The helpful comments of two anonymous referees are also gratefully acknowledged. This work was supported by the DFG.

\section{Abbreviations}

EPSP, excitatory postsynaptic potential; TC, tangential cell.

\section{References}

Bajjalieh, S.M. (1999) Synaptic vesicle docking and fusion. Curr. Opin. Neurobiol., 9, 321-328.

Bausenwein, B., Wolf, R. \& Heisenberg, M. (1986) Genetic dissection of optomotor behavior in Drosophila melanogaster. Studies on wild-type and the mutant optomotor-blind H31. J. Neurogenetics, 3, 87-109.

Borst, A. \& Haag, J. (1996) The intrinsic electrophysiological characteristics of fly lobula plate tangential cells: I. Passive membrane properties. J. Comp. Neurosci, 3, 313-336.

Calvin, W.H. \& Stevens, C.F. (1968) Synaptic noise and other sources of randomness in motoneuron interspike intervals. J. Neurophysiol., 31, 574587.

Collett, T.S. \& King, A.J. (1975) Vision during flight. In Horridge, G.A., (ed.), The Compound Eye and Vision of Insects. Clarendon Press, Oxford, pp. 437-466.

Dahmen, H.J., Wüst, R.M. \& Zeil, J. (1997) Extracting egomotion parameters from optic flow: Principal limits for animals and machines. In Srinivasan, M.V., Venkatesh, S. (eds), From Living Eyes to Seeing Machines. Oxford University Press, Oxford, New York, pp. 174-198.

Douglass, J.K. \& Strausfeld, N.J. (1996) Visual motion-detection circuits in flies: Parallel direction- and non-direction-sensitive pathways between the medulla and lobula plate. J. Neurosci, 16, 4551-4562.

Duffy, C.J. (1998) MST neurons respond to optic flow and translational movement. J. Neurophysiol., 80, 1816-1827. 
Egelhaaf, M. (1985) On the neuronal basis of figure-ground discrimination by relative motion in the visual system of the fly. I. Behavioural constraints imposed on the neuronal network and the role of the optomotor system. Biol. Cybern, 52, 123-140.

Egelhaaf, M. (1987) Dynamic properties of two control systems underlying visually guided turning in house-flies. J. Comp. Physiol. A, 161, 777-783.

Egelhaaf, M. (1989) Visual afferences to flight steering muscles controlling optomotor response of the fly. J. Comp. Physiol. A, 165, 719-730.

Egelhaaf, M. \& Borst, A. (1989) Transient and steady-state response properties of movement detectors. J. Opt. Soc. Am. A, 6, 116-127.

Egelhaaf, M. \& Borst, A. (1993a) A look into the cockpit of the fly: Visual orientation, algorithms, and identified neurons. J. Neurosci, 13, 4563-4574

Egelhaaf, M. \& Borst, A. (1993b) Movement detection in arthropods. In Wallman, J., Miles, F.A. (eds), Visual Motion and its Role in the Stabilization of Gaze. Elsevier, Amsterdam, London, New York, pp. 53-77.

Egelhaaf, M. \& Warzecha, A.-K. (1999) Encoding of motion in real time by the fly visual system. Curr. Opin. Neurobiol., 9, 454-460.

Egelhaaf, M., Borst, A., Warzecha, A.-K., Flecks, S. \& Wildemann, A. (1993) Neural circuit tuning fly visual interneurons to motion of small objects. II. Input organization of inhibitory circuit elements by electrophysiological and optical recording techniques. J. Neurophysiol., 69, 340-351.

Gaffron, M. (1934) Untersuchungen über das Bewegungssehen bei Libellenlarven, Fliegen und Fischen. Z. Vergl. Physiol., 20, 299-337.

Gauck, V., Egelhaaf, M. \& Borst, A. (1997) Synapse distribution on VCH, an inhibitory, motion-sensitive interneuron in the fly visual system. J. Comp. Neurol., 381, 489-499.

Geiger, G. \& Nässel, D.R. (1982) Visual processing of moving single objects and wide-field patterns in flies: Behavioural analysis after laser-surgical removal of interneurons. Biol. Cybern., 44, 141-149.

Haag, J. (1994) Aktive und passive Membraneigenschaften bewegungsempfindlicher Interneurone der Schmeissfliege Calliphora erythrocephala. Doctoral Dissertation. Universität Tübingen, Tübingen, Germany.

Haag, J. \& Borst, A. (1996) Amplification of high frequency synaptic inputs by active dendritic membrane processes. Nature, 379, 639-641.

Haag, J., Vermeulen, A. \& Borst, A. (1999) The intrinsic electrophysiological charateristics of fly lobula plate tangential cells: III. Visual response properties. J. Computat. Neurosci, 7, 213-234.

Hausen, K. (1976) Struktur, Funktion und Konnektivität bewegungsempfindlicher Interneurone im dritten optischen Neuropil der Schmeissfliege Calliphora erythrocephala. University of Tuebingen: Doctoral Dissertation. Universität Tübingen, Tübingen, Germany.

Hausen, K. (1981) Monocular and binocular computation of motion in the lobula plate of the fly. Verh. Dtsch. Zool. Ges, 74, 49-70.

Hausen, K. (1982a) Motion sensitive interneurons in the optomotor system of the fly. I. The horizontal cells: structure and signals. Biol. Cybern., 45, 143156

Hausen, K. (1982b) Motion sensitive interneurons in the optomotor system of the fly. II. The Horizontal Cells: Receptive field organization and response characteristics. Biol. Cybern, 46, 67-79.

Hausen, K. \& Egelhaaf, M. (1989) Neural mechanisms of visual course control in insects. In Stavenga, D., Hardie, R. (eds), Facets of Vision. Springer, Berlin,Heidelberg,New York pp. 391-424.

Hausen, K. \& Wehrhahn, C. (1983) Microsurgical lesion of horizontal cells changes optomotor yaw responses in the blowfly Calliphora erythrocephala. Proc. R. Soc. Lond. B, 219, 211-216.

Heide, G. (1983) Neural mechanisms of flight control in Diptera. In Nachtigall, W. (ed.), BIONA Report. Akademie der Wissenschaften und der Literatur zu Mainz and Gustav Fischer-Verlag, Mainz, Stuttgart, New York, pp. 35-52.

Heisenberg, M., Wonneberger, R. \& Wolf, R. (1978) Optomotor-blind - a Drosophila mutant of the lobula plate giant neurons. J. Comp. Physiol., 124, 287-296.

Hengstenberg, R., Krapp, H. \& Hengstenberg, B. (1998) Visual sensation of self-motion in the blowfly Calliphora. In Taddei-Ferretti, C. (ed.), Biocybernetics in Vision: Integrative and Cognitive Processes. World Scientific Press, Singapore.

Hertz, M. (1934) Zur Physiologie des Formen- und Bewegungssehens I. Optomotorische Versuche an Fliegen. Z. Vergl. Physiol., 20, 430-449.

Horstmann, W., Warzecha, A.-K. \& Egelhaaf, M. (1999) Synaptic transmission and postsynaptic integration in motion sensitive neurons of the fly (Calliphora). In Elsner, N., Eysel, U. (eds), Göttingen Neurobiology Report 1999, Thieme, Stuttgart, New York, p. 444.

Ibbotson, M.R. (1991) Wide-field motion-sensitive neurons tuned to horizontal movement in the honeybee, Apis mellifera. J. Comp. Physiol. A, 168, 91-102.
Ibbotson, M.R. \& Goodman, L.J. (1990) Response characteristics of four wide-field motion-sensitive descending interneurons in Apis mellifera. J. Exp. Biol., 148, 255-279.

Juusola, M., Uusitalo, R.O. \& Weckström, M. (1995) Transfer of graded potentials at the photoreceptor-interneuron synapse. J. Gen Physiol., 103, $117-148$.

Kern, R. (1998) Visual position stabilization in the hummingbird hawk moth, Macroglossum stellatarum L. II. Electrophysiological analysis of neurons sensitive to wide-field image motion. J. Comp. Physiol. A, 182, 239-249.

Kern, R. \& Varjú, D. (1998) Visual position stabilization in the hummingbird hawk moth, Macroglossum stellatarum L. I. Behavioural analysis. J. Comp. Physiol. A, 182, 225-237.

Kern, R., Nalbach, H. \& Varjú, D. (1993) Interactions of local movement detectors enhance the detection of rotation. Optokinetic experiments with the rock crab, Pachygrapsus marmoratus. Vis. Neurosci, 10, 643-652.

Koch, C. (1999) Biophysics of Computation. Oxford University Press, New York, Oxford.

Krapp, H.G. \& Hengstenberg, R. (1996) Estimation of self-motion by optic flow processing in single visual interneurons. Nature, 384, 463-466.

Krapp, H.G., Hengstenberg, B. \& Hengstenberg, R. (1998) Dendritic structure and receptive-field organization of optic flow processing interneurons in the fly. J. Neurophysiol., 79, 1902-1917.

Laughlin, S.B., de Ruyter van Steveninck, R. \& Anderson, J.C. (1998) The metabolic cost of neural information. Nature Neurosci, 1, 36-41.

Maddess, T. \& Laughlin, S.B. (1985) Adaptation of the motion-sensitive neuron $\mathrm{H} 1$ is generated locally and governed by contrast frequency. Proc. $R$. Soc. Lond. B, 225, 251-275.

Manor, Y., Nadim, F., Abbott, L.F. \& Marder, E. (1997) Temporal dynamics of graded synaptic transmission in the lobster stomatogastric ganglion. $J$. Neurosci, 17, 5610-5621.

Murthy, V.N. (1999) Optical detection of synaptic vesicle exocytosis and endocytosis. Curr. Opin. Neurobiol., 9, 314-320.

Nalbach, H.-O. (1992) Translating head movements of pigeons in repsonse to a rotating pattern: Characteristics and tool to analyse mechanisms underlying detection of rotational and translational optical flow. Exp. Brain Res., 92, 27-38.

Rind, F.C. \& Simmons, P.J. (1999) Seeing what is coming: building collisionsensitive neurones. Trends Neurosci, 22, 215-220.

Roberts, A. \& Bush, B.M.H. (1981) Neurones without impulses. Cambridge University Press, Cambridge, London. New York.

Simmons, P.J. (1999) The performance of synapses that convey discrete graded potentials in an insect visual pathway. J. Neurosci, 19, 1058410594.

Simpson, J.I., Leonard, C.S. \& Soodak, R.E. (1988) The accessory optic system of rabbit. II. Spatial organization of direction selectivity. $J$. Neurophysiol., 60, 2055-2072.

Strausfeld, N.J. (1976) Atlas of an insect brain. Springer, Berlin Heidelberg New York.

Strausfeld, N.J., Kong, A., Milde, J.J., Gilbert, C. \& Ramaiah, L. (1995) Oculomotor control in calliphorid flies: GABAergic organization in heterolateral inhibitory pathways. J. Comp. Neurol., 361, 298-320.

Strausfeld, N.J. (1989) Beneath the compound eye: Neuroanatomical analysis and physiological correlates in the study of insect vision. In Stavenga, D.G., Hardie, R.C. (eds), Facets of Vision. Springer, Berlin, Heidelberg, pp. 317 359.

Sun, H. \& Frost, B.J. (1998) Computation of different optical variables of looming objects in pigeon nucleus rotundus neurons. Nature Neurosci, $\mathbf{1}$, 296-303.

Uusitalo, R.O., Juusola, M., Kouvalainen, E. \& Weckström, M. (1995) Tonic transmitter release in a graded potential synapse. J. Neurophysiol., 74, 470473.

Warzecha, A.-K., Egelhaaf, M. \& Borst, A. (1993) Neural circuit tuning fly visual interneurons to motion of small objects. I. Dissection of the circuit by pharmacological and photoinactivation techniques. J. Neurophysiol., 69, 329-339.

Warzecha, A.-K., Kretzberg, J. \& Egelhaaf, M. (1998) Temporal precision of encoding of motion information by visual interneurons. Curr. Biol., 8, 359368.

Wylie, D.R., Bischof, W.F. \& Frost, B.J. (1998) Common reference frame for neural coding of translational and rotational optic flow. Nature, 392, 278282.

Wylie, D.R.W. \& Frost, B.J. (1999) Responses of neurons in the nucleus of the basal optic root to translational and rotational flowfields. J. Neurophysiol., 81, 267-276. 